\title{
Arterial pulse transit time and ECG-initiated transit time: The form of the conditioned responses
}

\author{
SELINA REDMAN \\ University of Newcastle, Newcastle, N.S.W., Australia \\ and \\ JOHN DUTCH \\ Victoria University, Wellington, New Zealand
}

\begin{abstract}
Arterial pulse transit time (PTT), ECG-initiated transit time (ECG-TT), and interbeat interval were recorded during a differential classical conditioning procedure with a commercial video game functioning as the unconditioned stimulus. In Experiment 1, subjects were presented with 20 acquisition trials. Both PTT and ECG-TT showed differential changes to the CS+ and CS-. The PTT CR took the form of a decrease in transit time, reaching maximum between 5 and 7 beats after CS onset, and the ECG-TT response was biphasic, consisting of an initial lengthening from baseline during the first three beats after CS onset followed by a decrease below baseline, reach ing maximum at Beats 8-10. Experiment 2 is a replication of Experiment 1 using 40 acquisition trials. PTT and ECG-TT CRs were similar to those observed in Experiment 1 and persisted during the 40 trials. The PTT decrease from baseline during Beats 5-7 was significant, as were the deceleratory and acceleratory ECG-TT components.
\end{abstract}

The study of classically conditioned cardiovascular response in human subjects has until recently been limited primarily to heart rate (e.g., Zeaman, Deane, \& Wegner, 1954) and vasomotor responding (e.g., Gottschalk, 1946). However, with technological developments, the recording of a wider range of cardiovascular responses has now become possible.

Redman and Dutch (1983) reported conditioned responding in two such new responses, arterial pulse transit time (PTT) and ECG-initiated transit time (ECG-TT). Using the cold pressor test as the unconditioned stimulus (UCS), a decrease in PTT was observed in response to the conditioned stimulus (CS) and a concomitant lengthening of ECG-TT occurred. However, since the cold pressor test is noxious and has long-lasting effects on the cardiovascular system, the number of acquisition trials was limited to eight. Therefore, a thorough investigation of the form and persistence of the PTT and ECG-TT conditioned responses (CRs) was not possible.

The purpose of the following experiments was to extend the findings of Redman and Dutch (1983) by exploring the form of the PTT and ECG-TT CRs and their persistence during extended acquisition. To avoid the

This research was supported by Grant 200/13279 from the New Zealand Scientific Research Distribution Committee, Grant 78/263 from the New Zealand University Grants Committee, and V.U.W. IRC,grants. Requests for reprints should be sent to $\mathrm{S}$. Redman, Discipline of Behavioural Science in Relation to Medicine, University of Newcastle, N.S.W. 2308, Australia. problems of the cold pressor, a video game was used as the UCS, since it has previously been found to elicit reliable cardiovascular changes (Dutch \& Redman, 1983).

\section{EXPERIMENT 1}

\section{Method}

Subjects. Ten university students, three female and seven male, aged between 18 and 30 years, volunteered to participate in the experiment.

Equipment and Physiological recording procedures. The presentation of stimuli and data collection were controlled by a Hewlett-Packard 9835 desktop computer. The physiological signals were processed through a Beckamn Type S Dynograph.

PTT was recorded as the interval between the detection of the pulse at the popliteal artery behind the left knee and its arrival at the dorsalis pedis artery on the upper surface of the left foot. The popliteal pulse was transduced using a Biocom piezo electric microphone and the dorsalis pedis pulse using a Narco Biosystems piezo electric Korotkoff sounds microphone. Both pulses were filtered at low-pass $30 \mathrm{~Hz}$, and then fed into a Gould Advance storage digital oscilloscope (OS 4000). PTT was calculated by the computer from the digitized output of the oscilloscope as the interval between the onset of the sweep (i.e., the $\mathrm{R}$ wave of the ECG signal) and the onset of the sharp upsweep of the popliteal pulse.

Interbeat interval (IBI) was measured between successive $\mathbf{R}$ waves of the ECG signal, using a Hewlett-Packard counter time with millisecond accuracy.

Stimuli. An Ohio Scientific C4-P computer presented the video game which was displayed on a Phillips 18 in. color TV screen immediately in the subject's line of vision. Timing of the presentation of the game and color stimuli by the C4-P was controlled by the Hewlett-Packard computer.

A commercial video game, OSI HECTIC, was modified for use as the UCS. In this game, the subject was required to operate a mobile spaceship. During the game, bombs fell in a random pattern from the 
top of the video screen and the subject's task was to destroy as many as possible. Movement and firing of the spaceship were operated by the depression of defined keys on the keyboard of the C4-P, requiring the use of only the right hand.

Following each game, the number of bombs destroyed on that trial was displayed for $5 \mathrm{sec}$ along with a running total of hits in the session overall. After each session, the total number of hits and the number of times the subject depressed the move and fire keys appeared on the screen.

Changes in the color (red or green) of the video screen functioned as the CS + and CS- . Between trials, the screen was black, and during the game, screen color was that of the CS+.

Procedure. All subjects attended for two identical sessions, during each of which $10 \mathrm{CS}+$ and $10 \mathrm{CS}$ - trials were presented in a random order. Each trial consisted of a 30-beat baseline period, a 10-beat CS + or CS - presentation, 60 beats of the video game on CS + trials, and a variable intertrial interval ranging from 50 to $110 \mathrm{sec}$.

Upon arrival at the laboratory, a subject was seated with his or her left leg in a horizontal position and supported under the heel, in a soundattenuated room separate from the recording equipment. The microphones and electrodes were attached to the subject, the game was briefly explained, and headphones, through which music was played throughtout the experiment, were positioned. The room was then darkened, and the subject was left alone for $5 \mathrm{~min}$ before data collection began.

\section{Results}

For each subject, and separately for each response, the median change from baseline on each beat during CS + and CS - presentation was calculated for every consecutive 5-trial block. The mean of these medians over all 20 trials was computed for each subject. Using these data, the mean and standard error for each beat across subjects was calculated; they are plotted in Figure 1. An inspection of the beat-by-beat changes during the CS was undertaken to provide a description of the form of the CRs.

In addition, for each response, the mean change from baseline during $\mathrm{CS}+$ presentations and the mean change from baseline during CS - presentations over the 20 trials was calculated for each subject. A two-tailed repeated measures $t$ test was run on these data to establish whether responding to the $\mathrm{CS}+$ differed from baseline or from responding during the $\mathrm{CS}-$ across subjects.

PTT. During CS + presentations, PTT decreased significantly from baseline $[\mathrm{t}(9)=3.18, \mathrm{p}<.02]$ and was significantly shorter than responding during the $\mathrm{CS}-[\mathrm{t}(9)$ $=3.83, \mathrm{p}<.01 \mathrm{~J}$. As is evident from Figure 1, the PTT response to the CS consisted of an initial sharp decrease in PTT, reaching maximum between 5-7 beats after CS onset, followed by a gradual return to baseline prior to UCS onset.

ECG-TT. During CS + presentations, ECG-TT was not significantly different from baseline $[t(9)=0.93$, n.s.] but was significantly shorter than responding during the $\mathrm{CS}-[\mathrm{t}(9)=2.35, \mathrm{p}<.05]$. The form of the ECG-TT response during CS + presentation consisted of an initial increase from baseline during the first 3 beats, followed by a decrese below baseline that was maximal during the last 3 beats before UCS onset.

IBI. During CS + presentations, IBI was not significantly different from baseline $[\mathrm{t}(9)=1.36$, n.s.] or from responding to the $\mathrm{CS}-[\mathrm{t}(9)=1.36, \mathrm{n} . \mathrm{s}$. $]$. In response to the CS +, IBI showed an initial sharp decrease from
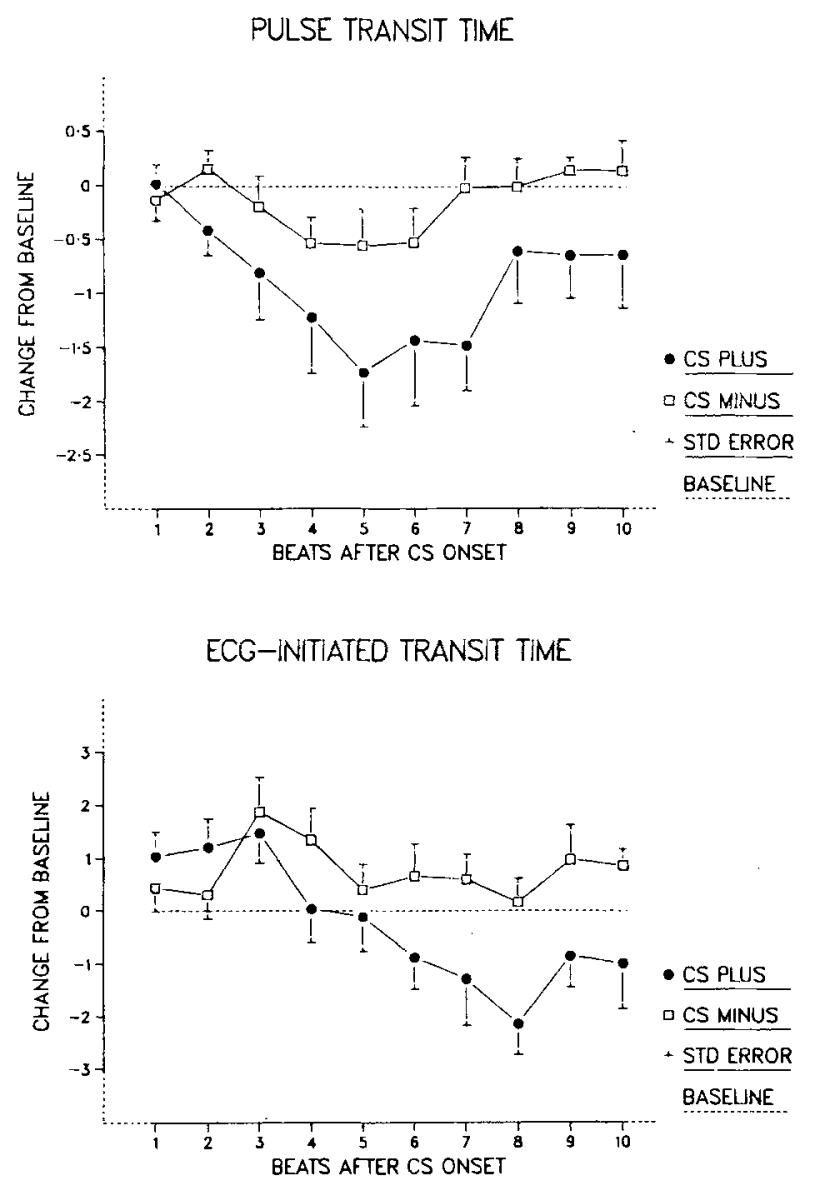

INTERBEAT INTERVAL

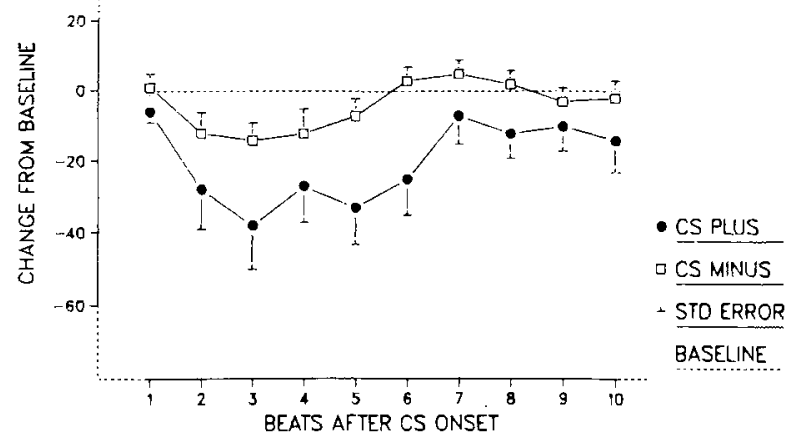

Figure 1. Experiment 1: Beat-by-beat changes in PTT, ECG-TT, and IBI during $\mathrm{CS}+$ and $\mathrm{CS}-$ presentation, as change from baseline in milliseconds.

baseline, reaching a minimum between 2 and 6 beats after CS onset, followed by a gradual return towards baseline prior to UCS onset.

\section{Discussion}

During 20 trials of classical conditioning with a video game as UCS, PTT and ECG-TT responses to the CS+ differed significantly from that to the $\mathrm{CS}-$. This experi- 
ment therefore extends earlier findings of PTT and ECGTT CRs (Redman \& Dutch, 1983), using a different stimulus as the UCS.

Examination of the form of the PTT and ECG-TT CRs indicated that the PTT response was a decrese from baseline that reached maximum between 5 and 7 beats after CS onset, whereas the ECG-TT CR was biphasic, consisting of an increase from baseline during Beats 1-3 and a decrease that reached maximum at Beats $8-10$ after CS onset. The IBI response to the CS took the form of a decrease from baseline, with the greatest change occurring between 2 and 6 beats after CS onset.

\section{EXPERIMENT 2}

The purpose of the following experiment was to replicate the findings of Experiment 1 and to further explore the forms of the conditioned PTT and ECG-TT responses. Based on the response form found in Experiment 1, Experiment 2 was designed to test the prediction that a conditioned PTT decrease would be observed between Beats 5 and 7 and that a biphasic ECG-TT with an increase from baseline during Beats 1-3 and a decrease from Beats 8-10 would be observed. To further explore the persistence of the CRs, PTT, ECG-TT, and IBI were recorded during 40 acquisition tirals.

\section{Method}

Subjects. Eight university students, seven male and one female, aged between 18 and 30 years, volunteered to participate in the experiment.

Procedure. Physiological recording procedures were identical to those described for Experiment 1.

All subjects attended for four sessions, which were identical to those of Experiment 1. Forty acquisition trials were given over these four sessions.

\section{Results}

Responding during the CS + was evaluated against baseline and against levels during CS - presentation separately for Trials 1-20 and Trials 21-40, in a manner similar to that described for Experiment 1.

Beat-by-beat changes to the CS + were also assessd in a similar manner to Experiment 1, but combined over all 40 acquisition trials. These response shapes are shown in Figure 2.

PTT. During CS + presentation, PTT decreased from baseline during both Trials 1-20 [t(7) $=3.19, \mathrm{p}<.02]$ and Trials $21-40[t(7)=2.95, \mathrm{p}<.05]$. PTT values decreased more from baseline during $\mathrm{CS}+$ than during $\mathrm{CS}-$, during the first $[\mathrm{t}(7)=4.42, \mathrm{p}<.01]$ and second $[\mathrm{t}(7)=4.54, \mathrm{p}<.01] 20$-trial blocks.

Figure 2 shows a response similar in shape to that observed in Experiment 1. For each subject, the mean PTT change from baseline during Beats 5-7 after CS onset was calculated. A two-tailed t test indicated a significant decrease from baseline on these beats across subjects $[t(7)$ $=3.80, \mathrm{p}<.01]$.

ECG-TT. ECG-TT did not significantly change from baseline during either the first $[t(7)=1.16, n . s$. $]$ or the
PULSE TRANSIT TIME

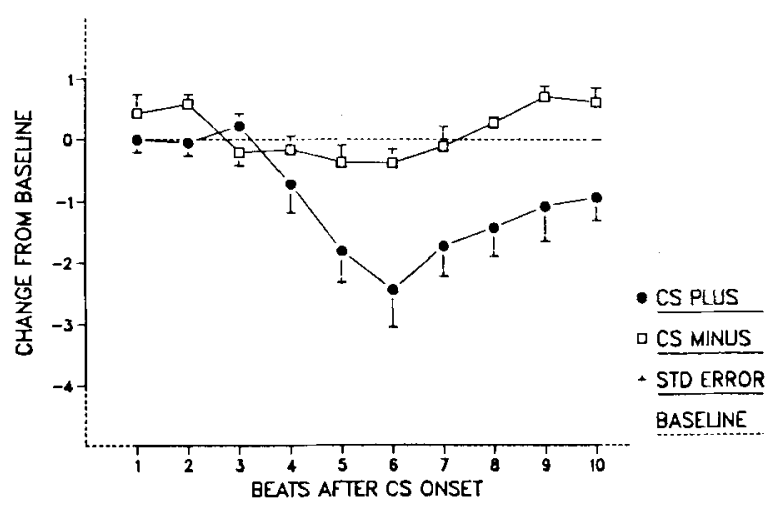

ECG-INTIIATED TRANSIT TIME

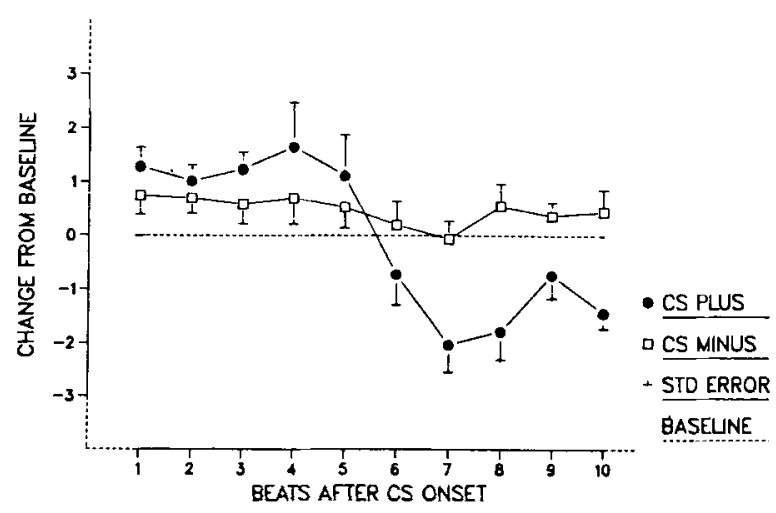

$|\mathrm{B}|$

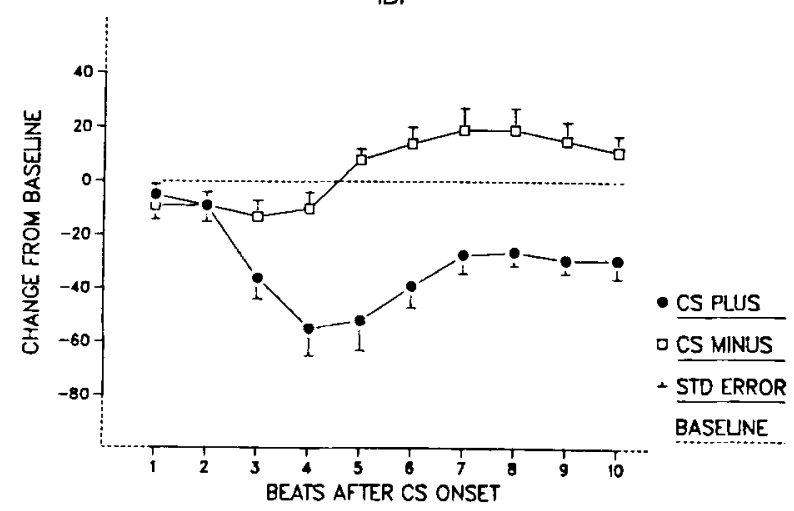

Figure 2. Experiment 2 Acquisition: Beat-by-beat changes in PTT, ECG-TT, and IBI during CS+ and CS - presentation, as change from baseline in milliseconds.

second [t(7) $=0.97$, n.s.] 20-trial block as a function of the biphasic response shape. Although there was no difference between the change from baseline during the CS + and the CS- over the first 20 trials, a greater decrease in ECG-TT occurred during CS + presentation during the second 20 trials $[\mathrm{t}(7)=5.71, \mathrm{p}<.001]$.

Figure 2 shows a ECG-TT response shape similar to that observed in Experiment 1. For each subject, the mean ECG-TT change from baseline was calculated separately 
during Beats 1-3 and during Beats 8-10. A two-tailed t test indicated that across subjects a significant lengthening of ECG-TT occurred during Beats 1-3 $[\mathrm{t}(7)=6.71$, $\mathrm{p}<.01]$. During Beats 8-10, ECG-TT significantly decreased from baseline $[\mathrm{t}(7)=3.63, \mathrm{p}<.01]$.

IBI. During CS + presentation, IBI decreased from baseline both during Trials 1-20 $[\mathrm{t}(7)=3.34, \mathrm{p}<.02]$ and during Trials $21-40$ [t(7) $=4.84, p<.01]$. IBI values decreased more from baseline during CS + presentation than during CS - during Trials $1-20[\mathrm{t}(7)=3.51$, $\mathrm{p}<.01]$ and during Trials 21-41 $[\mathrm{t}(7)=4.64$, $\mathrm{p}<.01]$.

An IBI response similar in shape to that observed during Experiment 1 occurred during CS + presentation, with IBI reaching the greatest change from baseline at Beat 3 after CS onset.

\section{Discussion}

Experiment 2 replicated the PTT and ECG-TT CRs observed in Experiment 1, and in addition indicated that these responses persist over 40 acquisition trials.

The PTT CR takes the form of a decrease in transit time which reaches a maximum between 5 and 7 beats after CS onset. Decreases in PTT occur as a function of increases in the rigidity of the artery wall (Bergel, 1961; Bramwell \& Hill, 1922). With a more rigid artery wall, there is a greater increase in pressure for the same increase in cardiac output, and flow during diastole is reduced (Berne \& Levy, 1977). Thus, increases in arterial rigidity will result in a greater workload on the left ventricle and indicate a less efficient performance at the periphery. In addition, PTT has been found to correlate with changes in diastolic blood pressure (Bramwell, McDowall, \& McSwiney, 1923; Geddes, Voelz, Babbs, Bourland, \& Tacker, 1981). The decreases in PTT observed in these experiments during CS+ presentation might therefore be argued to reflect both an increasing rigidity of the artery walls, which indicates less efficient cardiovascular functioning, and increases in diastolic blood pressure.

The ECG-TT CR took the form of an initial lengthening of transit time during Beats 1-3, followed by a decrease in the 3 beats prior to UCS onset. The ECG-TT is composed of two independent components, the preejection period, from the ECG signal to the ejection of blood into the aorta, and the transit time interval. As a function of changes in the duration of the preejection period component, ECG-TT has been shown to reflect changes in beta-adrenergic influences on the myocardium (Keller \& Rodbard, 1971; Obrist, Light, McCubbin, Hutcheson, \& Hoffer, 1979). ECG-TT has also been shown to correlate highly with systolic blood pressure (Allen, Schneider, Davidson, Winchester, \& Barr-Taylor, 1981) and with mean arterial pressure (Steptoe, Smulyan, \& Gribbin, 1976). During the presentation of the CS + in the experiments described here, the changes in ECG-TT appear to reflect an initial decrease in beta-adrenergic influence on the myocardium and in systolic blood pressure, followed by an increase in these aspects of cardiovascular activity prior to UCS onset.

The IBI CR is generally reported to consist of an initial acceleratory component, followed by a slowing below baseline prior to UCS onset (Obrist, Webb, \& Sutterer, 1969; Zeaman et al., 1954). In the present study, a similar initial acceleratory component was observed, but IBI remained faster than baseline during the entire CSUCS interval. The reason for the absence of a decelerative component with the procedure used here awaits future research.

In conclusion, the data from Experiments 1 and 2 provide a demonstration of classically conditioned responding in two previously unstudied cardiovascular responses, PTT and ECG-TT. The significance of the cardiovascular CRs occurring prior to the video game remain to be futher investigated, but it seems likely that they are similar to the preparatory cardiovascular responses observed during the foreperiod of a reaction time task (Obrist et al., 1969).

\section{REFERENCES}

Allen, R. A., Schneider, J. A., Davidson, D. M., Winchester, M. A., \& BARR-TAYLOR, C. (1981). The covariation of blood pressure and pulse transit time in hypertension patients. Psychophysiology, 18, 301-306.

BERGEL, D. H. (1961). The dynamic elastic properties of the arterial wall. Journal of Physiology (London), 156, 458-469.

BERNE, R. M., \& LEVY, M. N. (1977). Cardiovascular physiology (2nd ed.). St. Louis: Mosby.

Bramwell, J. C., Hill, A. V. (1922). The velocity of the pulse wave in man. Proceedings of the Royal Society of Biology, 93, 298-306.

Bramwell, J. C., McDowall, R. J. S., \& MCSwineY, B. A. (1923). Variation of arterial elasticity with blood pressure in man. Proceedings of the Royal Society of Biology, 94, 450-454.

DuTCH, J., \& REDMAN, S. (1983). Psychological stress and arterial pulse transit time. New Zealand Medical Journal, 96, 607-609.

Geddes, L. A., Voelz, M. H., BabBs, C. F., Bourland, J. D., \& TACKER, W. A. (1981). Pulse transit time as an indicator of arterial blood pessure. Psychophysiology, 18, 71-74.

GoTTSCHALK, L. A. (1946). A study of conditioned vasomotor responses in ten human subjects. Psychosomatic Medicine, 8, 16-27.

Keller, M. F., \& RodBaRD, S. (1971). Retardation of the arterial pressure wave by propranolol. American Heart Journal, 82, 794-795.

Obrist, P. A., Light, K. C., McCubbin, J. A., Hutcheson, J. S., \& HofFER, J. L. (1979). Pulse transit time: Relationship to blood pressure and myocardial performance. Psychophysiology, 16, 292-301.

Obrist, P. A., WebB, R. A., \& SutTerer, J. R. (1969). Heart-rate and somatic changes during aversive conditioning and a simple reaction time task. Psychophysiology, 5, 696-723.

REDMaN, S., \& Dutch, J. (1983). Classical conditioning of arterial pulse transit time and ECG-initiated transit time with the cold pressor as unconditioned stimulus. Physiological Psychology, 11, 130-134.

Steptoe, A., Smulyan, H., \& Gribin, B. (1976). Pulse wave velocity and blood pressure change: Calibration and applications. $P$ sychophysiology, 13, 488-493.

Zeaman, D., Deane, G., \& Wegner, N. (1954). Amplitude and latency characteristics of the conditioned heart rate response. Journal of Psychology, 38, 235-250.

(Manuscript received June 10, 1983; revision accepted for publication June 20,1984 .) 